Boise State University ScholarWorks

Materials Science and Engineering Faculty

Publications and Presentations

Department of Materials Science and Engineering

$3-1-2006$

\title{
Correlations Beyond the Nearest-Neighbor Level in Grain Boundary Networks
}

C. A. Schuh

Massachusetts Institute of Technology

M. Frary

Boise State University

\section{(c) $\oplus \Theta \Theta$}

This is an author-produced, peer-reviewed version of this article. (C) 2009, Elsevier. Licensed under the Creative Commons AttributionNonCommercial-NoDerivatives 4.0 International License (https://creativecommons.org/licenses/by-nc-nd/4.0/). The final, definitive version of this document can be found online at Scripta Materialia, doi: 10.1016/j.scriptamat.2005.11.044 


\title{
Correlations Beyond the Nearest-Neighbor Level in Grain Boundary Networks
}

\author{
C. A. Schuh and M. Frary \\ Department of Materials Science and Engineering, Massachusetts Institute of Technology \\ Cambridge, MA 02139 USA
}

Keywords: Grain Boundaries, Triple Junctions, Percolation

\begin{abstract}
Correlations among 'special' and 'general' grain boundaries are studied on two-dimensional networks, by examining the configurational entropy of boundary structures as well as percolation thresholds. Consideration of crystallographic constraints at various length scales reveals that higher-order constraints play a role in boundary connectivity and network structure. Implications for grain boundary engineering are discussed and directions for future work highlighted.
\end{abstract}

\section{Introduction}

The motivation behind grain boundary engineering is that grain boundaries exhibit a wide spread in their physical properties, and not all boundaries need be regarded as detrimental for processes of intergranular degradation or failure. In this context, it is relatively common to divide grain boundaries in a binary fashion into 'general' vs. 'special' (i.e., damage susceptible vs. damage resistant) types. This approach has proven extremely useful for understanding the structure of the grain boundary network, as it lends itself to analysis by the methods of percolation theory, where the grain boundary network is modeled as a bond lattice [1-4]. The fraction of special boundaries then becomes the important microstructural state variable that controls boundary clustering, and the percolation threshold for general boundaries becomes a natural 'target' for the grain boundary engineer: materials with special fractions above the threshold are, in principle, not prone to long-range intergranular damage.

In the ongoing effort to develop percolation theory for grain boundary networks, one major focus has been on the local correlations among special and general grain boundaries in the network. These correlations were first observed experimentally at the points of nearest-neighbor connectivity, the triple junctions $[5,6]$, and explained on the basis of crystallography $[7,8]$. The fact that correlations are present in grain boundary networks is of great significance in grain boundary engineering, because the clustering behavior and percolation threshold are substantially different from expectations based on random bond percolation problems [5, 8, 9].

Although nearest-neighbor correlations among grain boundaries are now reasonably well understood, a clear direction for future work in this field is to measure and understand longer-range correlations. Several authors have speculated that longer-range correlations may exist [10-12], but apart from our most recent study on quadruple junction character [9], there has been no quantitative exploration of this issue. In this note, we offer the first study of correlations at the second and third nearest-neighbor levels in two-dimensional (2D) grain boundary networks, and point to critical issues for the development of a comprehensive percolation theory for grain boundary networks.

\section{Methods}

We simulate two-dimensional grain boundary networks on ideal honeycomb lattices by first assigning grain orientations (three Euler angles, for grains of assumed cubic symmetry) and subsequently calculating boundary misorientations. Three 'families' of microstructures have been simulated, each of which is produced with a different method for selecting the initial grain orientations. These are described as:

a) 'General Textured' microstructures, which range from an ideal single-component texture to ideally random. In this case, special boundaries are of the low-angle variety (with disorientations below $15^{\circ}$ ), and are promoted by sharpening the texture.

b) 'Fiber Textured' microstructures, which range from ideal single-component textures to ideal 'ring' fiber textures, and again where special boundaries are of the lowangle variety.

c) 'Twinned' microstructures, in which all grain orientations are related to one another by $\Sigma 3^{\mathrm{n}}$ rotations, and where special boundaries are coincidence boundaries with 
$\Sigma \leq 29$ and lie within the Brandon criterion [13].

More details on the general simulation procedures are available in Refs. [8, 9], and are omitted here for brevity. Once constructed, the networks are analyzed in terms of their special boundary fractions, as well as the connectivity among boundary types.

\section{Analysis of Boundary Correlations}

Our study of correlations in the grain boundary network is based upon our understanding of crystallographic constraints that are present in any microstructure, and which restrict the way in which grain boundary types may be assembled into a network. These constraints are formally expressed by the need for orientation conservation around a Frank-Nabarro circuit through the microstructure; the misorientations around any closed loop must be selfcompensating, so that the beginning and ending of the circuit have the same orientation. The simplest possible non-trivial circuit of this kind is that which encircles a triple junction (see, for example, Fig. 1a); this is referred to as a constraint of first order, and the boundary correlations that arise from this constraint are already well understood [7, 8]. In this work we proceed to examine higher-order constraints, which represent larger Frank-Nabarro circuits that traverse more grains and grain boundaries. In general, we will identify the order of the constraint, $N$, with the number of triple junctions encircled by the circuit; Fig. 1 illustrates the first three orders of constraint for a 2D honeycomb network.

Following upon prior work in the field, boundary correlations are quantified through examination of local statistics. At the first-order level there are four topologically unique species of triple junctions as shown in Fig. 1, and their statistics represent the now common 'triple junction distribution' [5, 6, 14-17]. A similar statistical analysis is possible for the higher-order circuits in Fig. 1, although the analysis becomes considerably more complicated due to the rapidly increasing number of unique species (called $D$, and specified below each unit in Fig. 1). For example, at the thirdorder level there are $D=72$ unique species (allowing for mirror-symmetric redundancy), as drawn explicitly in Fig. 1.

To proceed, we would like to quantitatively evaluate the 'strength' of each constraint shown in Fig. 1. For this purpose we will use the configurational entropy, $S$, of the largest, third-order units:

$$
S=-\sum_{i} f_{i} \ln f_{i}
$$

where $f_{\mathrm{i}}$ is the fraction of the $i^{\text {th }}$ species from among the $D=72$ species drawn in Fig. 1. Entropy is chosen as a metric for grain boundary correlations because constraints usually increase the information content (and reduce the disorder) in the system. The calculation is performed for the third-order boundary structure simply because this structure contains information about all of the lower-order constraints. In fact, every circuit in Fig. 1 necessarily contains within it smaller loops of lower order, but in general, the higher-order circuits involve additional, nonredundant constraints because they encircle some boundaries which they do not cross. In the case of the third-order boundary structure there are three subcircuits around the triple junctions (first-order constraints), as well as two second-order circuits. None of these is necessarily redundant with one another or with the third-order constraint, and the important question as we proceed is: how can we deconvolve the individual contributions of each constraint to the configurational entropy, $S$ ?

Our procedure to extract the several entropic contributions is established in detail for a complementary case in three dimensions in Ref. [9]. There the second-order constraint around quadruple nodes was evaluated from the first-order triple junction constraint, and the entropy change induced by each was extracted. Here we use the same general procedure: the probability of finding a structure of third order is calculated using a straightforward probabilistic calculation based on the statistics of the elements of a lower order, $N$. Repeating this procedure for $N=1$ and 2 gives expectations for the population of third-order species if only constraints up to $N^{\text {th }}$ order are enforced. We define, therefore, individual entropy increments in the following way:

$\Delta \mathrm{S}_{\mathrm{N}}^{3}=\left|\mathrm{S}_{3}^{3}-\mathrm{S}_{\mathrm{N}}^{3}\right|$

where $S_{i}^{j}$ is the entropy among units of order $j$, given complete crystallographic constraints up to order $i$. In this work we will only examine entropy calculated at the $j=3$ level, although the concept is easily extended to higher (or lower) orders.

Based on these calculations, we can examine how the entropy of the $N=3$ boundary structures (shown in Fig. 1c) evolves as constraints are added in order from least to greatest. Furthermore, we will also compare to the $N=0$ case, which is the unconstrained case where boundaries are simply assigned at random. In what follows, we present only graphical results of these calculations, and suppress hundreds of lengthy statistical equations which can all be easily reproduced using the method of Ref. [9], and which are collected elsewhere for the interested reader [18].

\section{Entropy and Constraint}

To begin our discussion, we first examine the magnitude of the total entropy change that occurs when all crystallographic constraints up to $N=3$ are 
imposed on an initially random network. This quantity is $\Delta \mathrm{S}_{0}^{3}$, and is plotted in Fig. 2 for the three microstructural families, in each case as a function of the special boundary fraction, $p$. There are two important points conveyed by this figure. First, the level of constraint in grain boundary networks may vary significantly with the special fraction; grain boundary engineering to increase $p$ may lead to fundamental changes in grain boundary correlations. Second, Fig. 2 also shows that different microstructural families can have considerably different correlations, with the fiber textured class in this case exhibiting more significant entropy changes due to crystallographic constraint. This result is in line with prior results on these simulated microstructures [8, 9], which showed that fiber textured materials have the strongest nearestneighbor correlations in both $2 \mathrm{D}$ and $3 \mathrm{D}$.

Although Fig. 2 focused upon the total entropy change given complete crystallographic constraint out to third order, $\Delta S_{0}^{3}$, similar plots can be constructed for each of the individual contributions to this total entropy. Rather than examine all of these curves individually, we instead focus upon the behavior in the vicinity of the general boundary percolation threshold ( $p \approx 0.35$ ), because this is the point where correlations have the most impact on network structure and therefore materials properties. In Fig. 3, we explicitly plot the contribution of each constraint, and examine the entropy change resulting from each. For all of the curves in Fig. 3, we see that the highest entropy is associated with $N=0$ (i.e., a random network without constraint), and the progressive addition of constraints at $N=1,2$ and 3 leads to a decrease in the system entropy. Furthermore, the largest drop in entropy always occurs at the first-order level; this is the triple junction constraint studied previously in the literature, which we see here is usually dominant in dictating the system entropy. For example, although the fiber textured microstructures have the largest values of $\Delta S_{0}^{3}$ in Fig. $3, \Delta S_{1}^{3}$ in these microstructures approaches zero, suggesting that first-order constraints alone are responsible for virtually all of the information in the system. However, we also clearly see that higher-order constraints are not always negligible; the entropy drop upon addition of second-order constraints (from $N=1$ to $N=2$ ) in general textured and twinned microstructures is still clearly non-zero. In these microstructural families, it seems that higher-order constraints are relatively more important. To our knowledge, the significance of longer-range correlations has not been appreciated in any prior work on the structure of grain boundary networks. In fact, these effects cannot be observed through studies of, e.g., the triple junction distribution, which samples only first-order effects.

\section{Percolation Thresholds}

One link between grain boundary network structure and properties is through the percolation threshold, which, in a single number, gives information about connectivity over large length scales. It is well known that finite-scale correlations shift the percolation threshold, and this issue has been explored in grain boundary networks in prior work $[4,5,8,9]$. Here we decouple the effects of first- and higher-order constraints upon the percolation thresholds of a 2D honeycomb lattice, as illustrated in Fig. 4. Large networks (larger than 300 x 300 grains) have been simulated using either (i) a process of random grain boundary character assignment, (ii) a process of triple junction assignment incorporating first-order constraints, or (iii) completely crystallographically-consistent assignments of grain orientation. The percolation thresholds of these networks were found to within \pm 0.005 , using many discrete simulations analyzed with the standard Hoshen-Kopelman algorithm [19]. The thresholds for both general and special grain boundaries were identified, and are differentiated in Fig. 4 by the closed and open data points, respectively.

Looking first at the points for the fiber textured microstructural family, we see that the percolation threshold shifts significantly when firstorder constraints are imposed upon the system, but that additional higher-order constraints do relatively little to change the picture. This result is consistent with our prior observations from Fig. 3, where we found a dominant first-order constraint in these microstructures. In contrast, the percolation thresholds in both the general textured and twinned microstructures vary with each constraint imposed on the system. Interestingly, the higher-order constraints seem to have a relatively large impact on the threshold, and in fact, cause a shift in the opposite direction as compared to the first-order constraint. This result is probably related to the earlier observation from Fig. 3 that these microstructural families have significant higher-order constraints, and we now see that these can actually have very different influences on the network structure as compared to the triple junction constraint.

\section{Discussion and Conclusions}

The calculations presented in this work represent the first systematic exploration of higherorder constraints in grain boundary networks, and reveal some intriguing directions for future inquiry. One point that emerges by looking at configurational entropy as well as the percolation thresholds is that 
higher-order constraints cannot, in general, be neglected when studying the connectivity of grain boundary networks. The state-of-the-art in experimental correlation analysis at present is the triple junction distribution; we now suspect that this metric alone is insufficient for a complete understanding of network structure and prediction of properties. Furthermore, we have seen here a great complexity in the way constraints of different order influence the network structure. In particular, constraints of different order may actually compete with one another to shift the percolation threshold up or down in $p$ (c.f., Fig. 4), although here this is demonstrated only in the particular case of 2D honeycomb lattices.

Another point of particular concern is the significant differences seen from one family of microstructures to the next; whereas polycrystals sharing a common crystallographic axis (fiber textured family) seem to have only very short-range correlations, more complex textures induce longerrange correlations out to at least third order and possibly beyond. We believe this may be related to the details of the crystallographic constraint equations. When the crystals share a crystallographic axis as in our fiber textured materials, it is known that the first-order constraint is rigid (the three signed boundary disorientations sum exactly to zero) [8]. In this case, higher order constraints are, to a large extent, redundant. In contrast, for general textured and twinned microstructures the first-order constraint is much less rigid, so higher-order constraints can provide significantly more information content. The quantitative details as to how crystallographic texture influences grain boundary correlations are certainly not clear at present, and this represents a key issue for the future generalization of percolation theory to any grain boundary network.

To conclude this note, we point out that thorough analytical calculations of grain boundary constraints, like those performed here, are rather complex. Already at the third-order level we have had to consider the statistics of $D=72$ structural units. Beyond the third order, additional topological complexities arise because there are non-redundant conformational variations of the Frank-Nabarro circuit. For example, we identify three unique conformations of the fourth-order circuit (see Fig. 5a), the statistics of which must be considered separately. Fig. 5b shows that there are yet more unique circuits at $N=5$; it is easy to see how the number of species involved in entropy calculations quickly becomes too large to handle analytically. For this reason, we believe that the study of medium- and long-range structure in grain boundary networks may best be addressed through, e.g., the use of scaling laws [20].

\section{Acknowledgements}

This work was supported by the National Science Foundation under Contract \#DMR-0346848, although the views expressed here are not endorsed by the sponsor.

\section{References}

[1] Gertsman VY, Tangri K. Acta Mater. 1997; 45: 4107.

[2] Pan Y, Olson T, Adams BL. Can. Metall. Q. 1995; 34: 147.

[3] Wells DB, Stewart J, Herbert AW, Scott PM, Williams DE. Corrosion 1989; 45: 649.

[4] Frary M, Schuh CA. Appl. Phys. Lett. 2003; 83: 3755.

[5] Schuh CA, Minich RW, Kumar M. Phil. Mag. 2003; A83: 711.

[6] Kumar M, King WE, Schwartz AJ. Acta Mater. 2000; 48: 2081.

[7] Minich RW, Schuh CA, Kumar M. Phys. Rev. B 2002; 66: 052101.

[8] Frary M, Schuh CA. Phys. Rev. B 2004; 69: 134115.

[9] Frary M, Schuh CA. Phil. Mag. 2005; 85: 1123.

[10] Beran MJ, Mason TA, Adams BL, Olsen T. J. Mech. Phys. Solids 1996; 44: 1543.

[11] Reed BW, Minich RW, Rudd RE, Kumar M. Acta Crystallogr. 2004; A60: 263.

[12] Gertsman VY. Acta Crystallogr. 2001; A57: 649.

[13] Brandon DG. Acta Metall. 1966; 14: 1479.

[14] Schuh CA, Kumar M. J. Mater. Sci. 2005; 40: 847.

[15] Fortier P, Miller WA, Aust KT. Acta Mater. 1997; 45: 3459.

[16] Davies P, Randle V, Watkins G, Davies H. J. Mater. Sci. 2002; 37: 4203.

[17] Randle V, Davies P. Interface Sci. 1999; 7: 5.

[18] Frary M, Crystallographically Consistent Percolation Theory for Grain Boundary Networks. 2005, Ph.D. Thesis, Department of Materials Science and Engineering, Massachusetts Institute of Technology, Cambridge, MA.

[19] Hoshen J, Kopelman R. Phys. Rev. B 1976; 14: 3438.

[20] Frary M, Schuh CA. Acta Mater. (in print) 2005. 
(a)

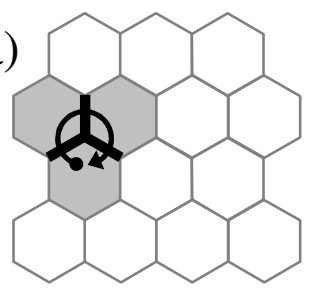

$\mathrm{N}=1$

$\mathrm{D}=4$ (b)

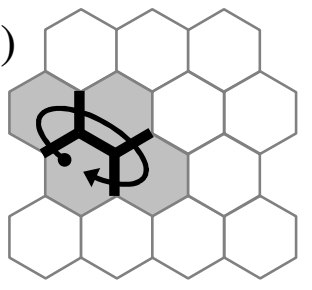

$\mathrm{N}=2$ (c)

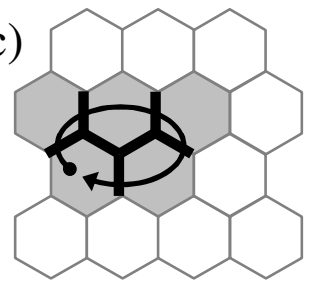

$\mathrm{N}=3$

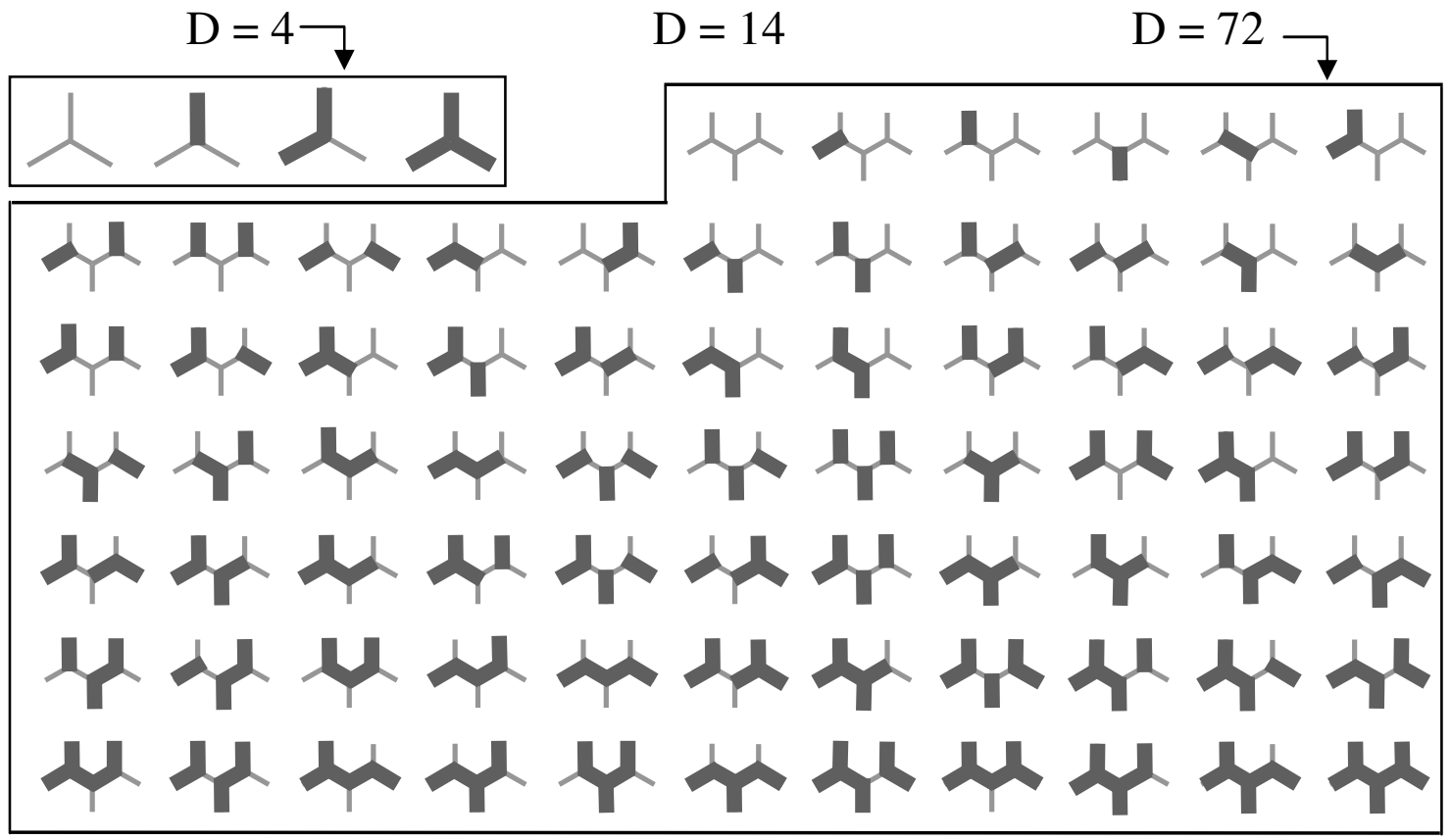

Figure 1: The first three orders of constraint in 2D honeycomb lattices. The order of the constraint, $N$, is equal to the number of triple junctions encircled by the Frank-Nabarro circuit. The number of topologically unique species of each order, $D$, is identified below each circuit as well. For $N=1$ and $N=3$, a representative structure is shown for each of the unique species in which the thinner lines indicate general boundaries and the thicker lines special boundaries. 


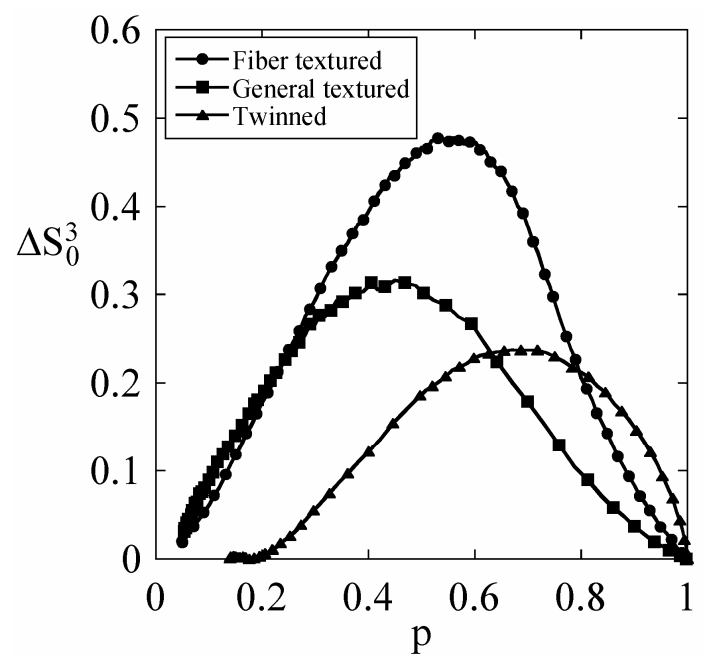

Figure 2: The magnitude of the total entropy change between a randomly assembled network and one in which full crystallographic constraints are imposed, plotted as a function of $p . \Delta \mathrm{S}_{0}^{3}$ is calculated from Eq. 2 using the population of $N=3$ boundary structures.

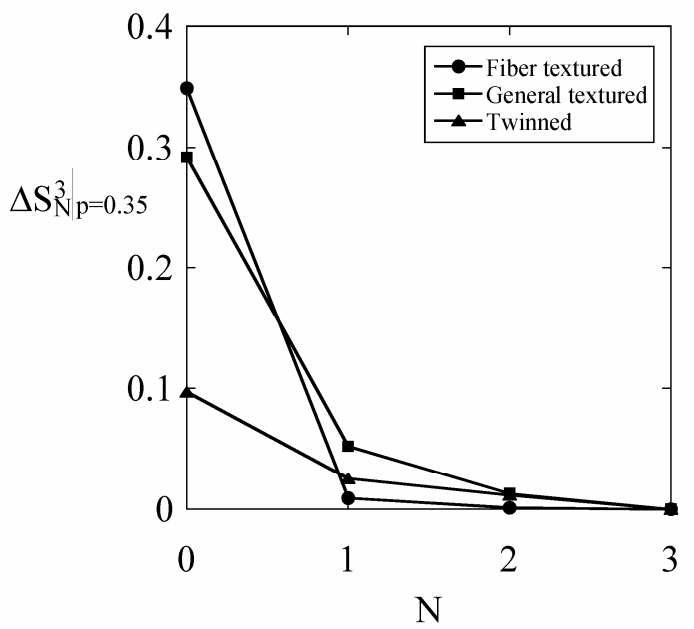

Figure 3: The contribution of each constraint level $N$ to the total change in configurational entropy, $\Delta \mathrm{S}_{\mathrm{N}}^{3}$, evaluated at $p=0.35$ for the $N=3$ boundary structure. 


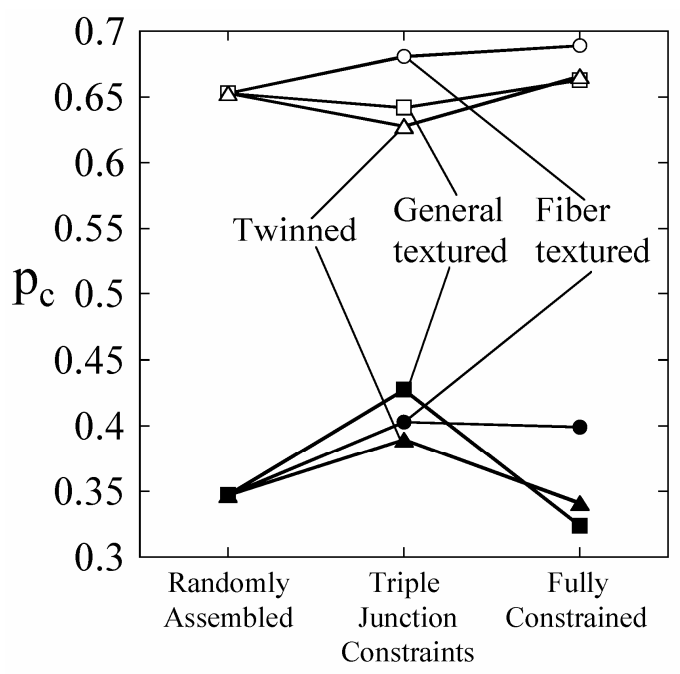

Figure 4: The percolation thresholds for 2D honeycomb lattices of grain boundaries; thresholds for special boundaries (open symbols) and general boundaries (filled symbols) are shown as a function of the constraints imposed on the system. Left to right, these data correspond to networks that were simulated using a process of (i) random grain boundary character assignment (no constraints imposed), (ii) triple junction assignment (only firstorder constraints imposed), or (iii) grain orientation assignment (full crystallographic constraints imposed).

(a) $\mathrm{N}=4$
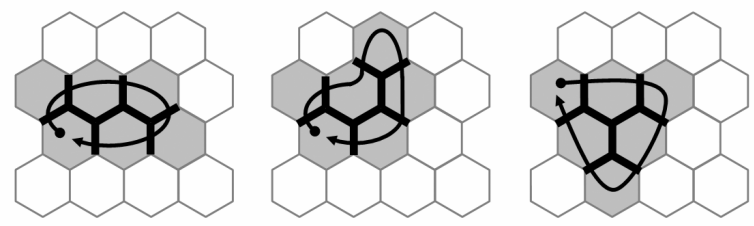

(b) $\mathrm{N}=5$
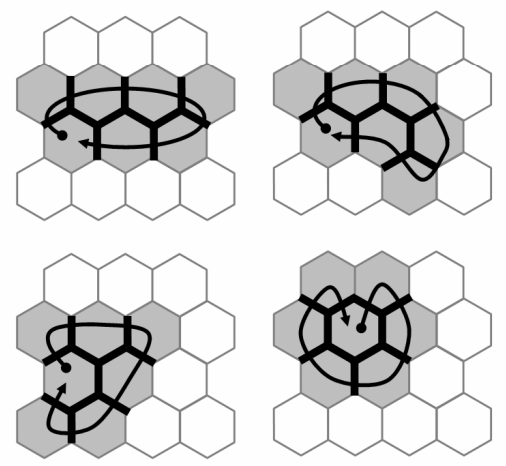

Figure 5: Frank-Nabarro circuits of fourth (a) and fifth (b) order. 